\title{
The substitution of glycine 661 by arginine in type III collagen produces mutant molecules with different thermal stabilities and causes Ehlers-Danlos syndrome type IV
}

\author{
Allan Richards, Paolo Narcisi, Jan Lloyd, Carole Ferguson, F Michael Pope
}

\begin{abstract}
Previous studies have shown that EhlersDanlos syndrome type IV (EDS IV) is caused by mutations of type III collagen (COL3A1). Here we have characterised the most amino-terminal glycine substitution so far described in a patient with EDS IV. A combination of peptide mapping and chemical cleavage analysis of cDNA localised the mutation in cyanogen bromide peptide CB5. Sequence analysis showed a $G$ to $A$ mutation, converting glycine 661 to arginine, which was a new dominant mutation. Analysis of type III collagen secreted by cultured fibroblasts showed an overmodified mutant protein with normal thermal stability. However, the intracellularly retained form melted $2^{\circ} \mathrm{C}$ lower than normal. This indicated that molecules resulting from the same mutation can differ in their thermal stabilities.
\end{abstract}

(f Med Genet 1993;30:690-3)

Ehlers-Danlos syndrome type IV (EDS IV) is the most severe of a heterogeneous group of disorders and is usually inherited in an autosomal dominant manner. ${ }^{1}$ Affected patients lack or have reduced amounts of type III collagen in their skin. ${ }^{2}$ Their cultured skin fibroblasts also secrete a reduced amount of this molecule, mostly retaining the mutant form intracellularly. ${ }^{3}$ A number of mutations in the gene (COL $3 \mathrm{~A} 1$ ) encoding this protein have been found, which include the substitution of glycine residues. ${ }^{4}$

The presence of a glycine at every third amino acid is a prerequisite for the formation of a stable collagen helix. Thus the substitution of a glycine in one of these positions can destabilise the helix and delay its formation, causing excessive post-translational modification N-terminal to the point of the substitution. Analysis of such overmodification has proved useful in mapping the site of single amino acid mutations in collagen molecules. ${ }^{56}$

In type III collagen these amino acid substitutions have produced various effects on the stability of the molecule as judged by thermal induction of helix to coil transition. These effects range from a 2 to $4^{\circ} \mathrm{C}$ reduction in thermal stability ${ }^{7}$ to a normal melting temperature. ${ }^{8}$ Here we provide evidence that a single amino acid substitution can produce mutant molecules which differ in their thermal stabilities.

\section{Materials and methods}

The full clinical details of the patient have been reported previously by Pope et $a l^{9}$ (patient 1). She is a non-acrogeric case of EDS IV and is the only affected member of her family.

Dermal fibroblasts were cultured, metabolically labelled, and procollagens and collagens analysed as previously described. ${ }^{10}$ Cyanogen bromide peptide mapping of type III collagen was also as previously described. ${ }^{6}$

Total cytoplasmic RNA was isolated from cultured dermal fibroblasts by lysing the cells with NP40 in the presence of vanadyl ribonucleoside complex. First strand synthesis was performed as previously described ${ }^{11}$ using the antisense primers described below.

The cDNA encoding CB9 or CB5 was amplified using the oligonucleotide primers CB93, CB95, ${ }^{11}$ CB53, and CB55 ${ }^{12}$ under conditions previously described. ${ }^{11}$ Alternatively the primer $\mathrm{E} 37^{12}$ was used in conjunction with CB53 to synthesise cDNA encoding the Cterminal half of CB5.

Chemical cleavage analysis was performed as previously described ${ }^{11}$ to detect mutations in cDNA encoding CB9 and CB5.

Analysis of DNA obtained from the proband's blood leucocytes was performed using the restriction enzyme Eco47III. After amplification with the oligonucleotide primers E36:5'GGTGAGAGAGGTGAAACTGGC3'sense and AVECO47:5'GGGTCCTGCAACTCCAGGAGGGCCTCCTTCACCTTTCTCACCCGGAGCGC3'-antisense, which were in exon 36 and 37 respectively, DNA was incubated with the enzyme at $37^{\circ} \mathrm{C}$ for 16 hours and analysed by agarose gel electrophoresis.

The thermal stability of type III collagen synthesised by the proband's cultured fibroblasts was as previously described. ${ }^{13}$

\section{Results}

Initial protein analysis of the labelled procollagens and collagens synthesised by the proband's cultured fibroblasts was performed by SDS-PAGE. The pro $\alpha$ (III) collagen was poorly secreted (data not shown). After treatment with pepsin, the type III collagen was analysed by electrophoresis with delayed reduction (fig 1A). An extra protein was visible in the proband's sample, migrating slightly above the normal $\alpha 1$ (III) collagen chains. A similar sized protein was also retained intracel- 


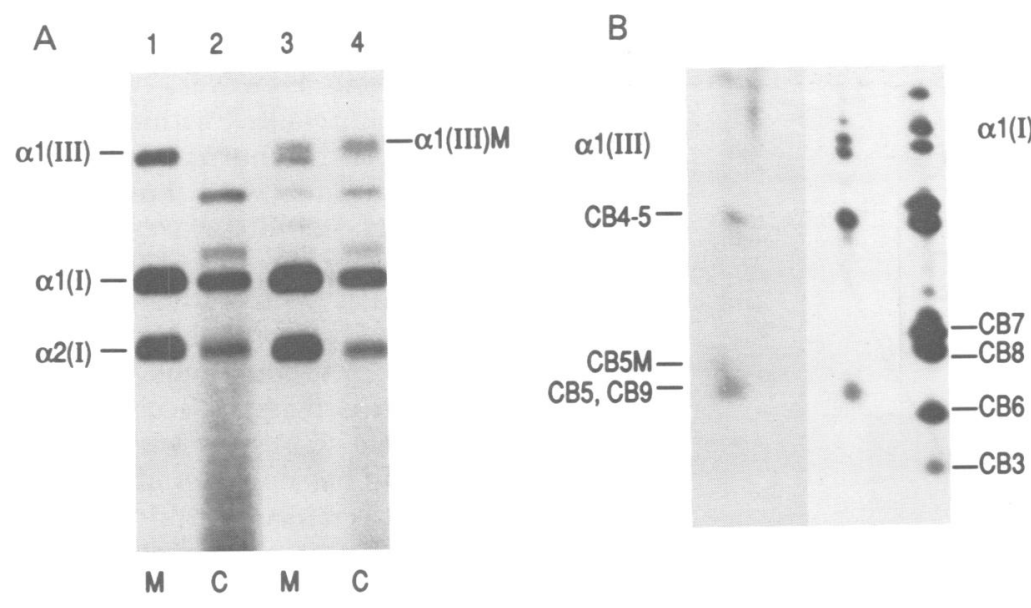

C

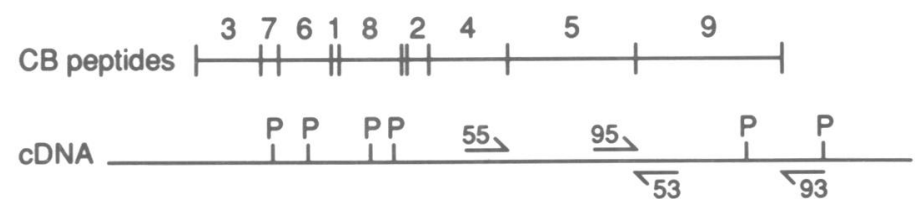

Figure 1 Protein analysis. SDS-PAGE analysis of $(A)$ radiolabelled collagens secreted into the medium $(M)$ or retained within the cell layer $(C)$ isolated from a normal control (lanes 1 and 2) and the proband (lanes 3 and 4). Normal ( $\alpha 1$ (III)) and slow $(\alpha 1(I I I) M)$ migrating type III collagens are shown, as are the two type I collagen $\alpha$ chains $(\alpha 1(I)$ and $\alpha 2(I)$ ). (B) Type III collagen cyanogen bromide peptides ( $\alpha 1(I I I) C B 5, C B 9, C B 4-5)$ from the proband (lane 1) and a normal control (lane 2). The slow migrating CB5 component is indicated as CBSM.

Cyanogen bromide peptides of the $\alpha 1$ (I) collagen (lane 3) was used as a standard. (C) The arrangement of $C B$ peptides of type III collagen. The position of the oligonucleotides $C B 93, C B 95, C B 53$, and $C B 55$ are indicated. PstI restriction sites are indicated by $P$
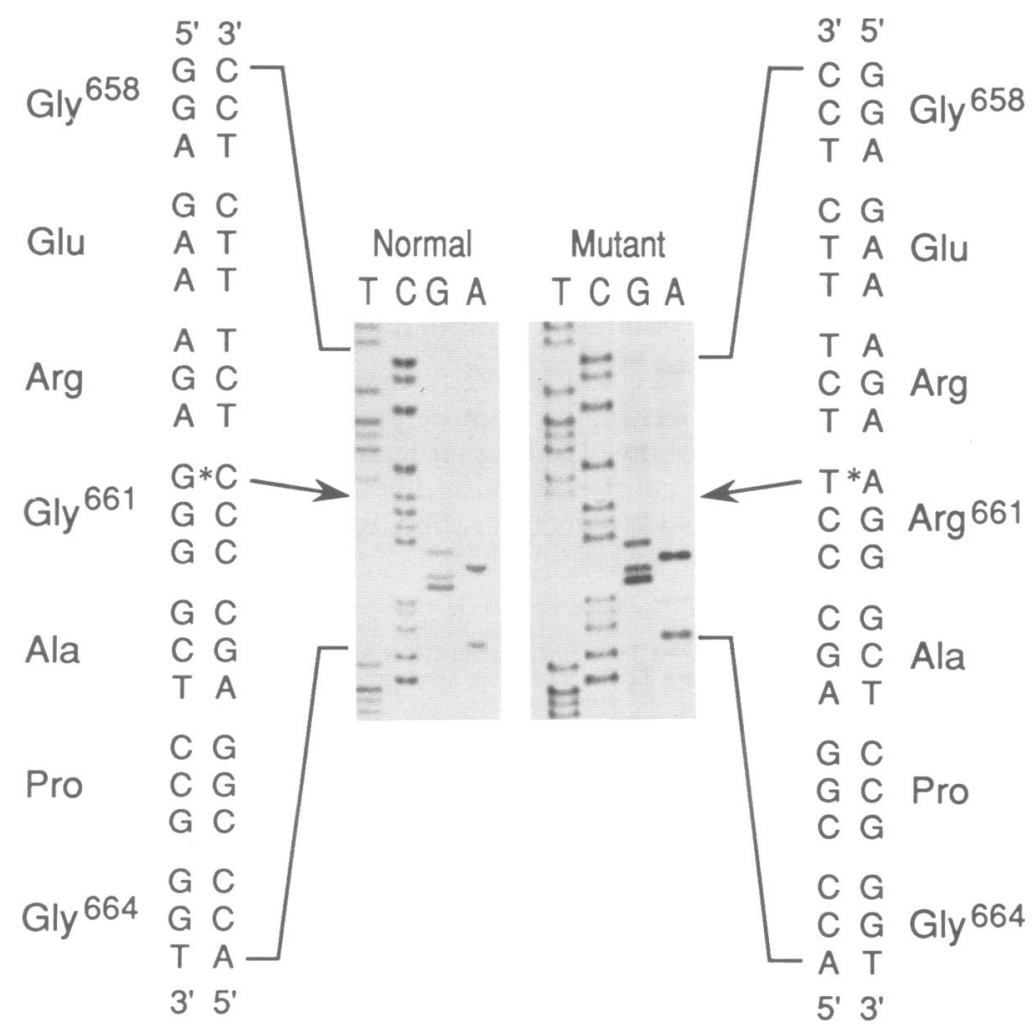

Figure 2 cDNA sequences. Antisense sequences from a normal and mutant clone are shown. The sense sequence is written adjacent to the sequences obtained from the gel. The mutated base is indicated by the arrows. lularly. This was consistent with an overmodified form of the protein caused by a mutation being present. These two species of type III collagen were excised from the gel, cleaved in situ with cyanogen bromide, and then subjected to re-electrophoresis in a second SDSpolyacrylamide gel (fig 1B). Analysis of the pattern showed a spot in the normal position of CB5 and CB9 and one spot migrating slightly slower than the normal products. This contrasted with a previously characterised mutation in CB9, ${ }^{6}$ where two abnormally migrating spots were seen. Thus, here, only CB5 appeared to be overmodified while CB9 was normal. Since overmodification normally occurs $\mathrm{N}$-terminal to the site of a mutation, this suggested that a glycine substitution had occurred in CB5 (fig 1C). This was confirmed by chemical cleavage mapping of cDNAs encoding either CB5 or CB9. Whereas a $339 \mathrm{bp}$ cleavage product was detected using the CB5 cDNA, none was detected using the cDNA for CB9 (data not shown). This analysis estimated the site of the mutation to be close to base 2591 of the complete cDNA sequence of type III collagen as described in the EMBL DNA sequence data base, accession number $\mathrm{X} 14420{ }^{14} \mathrm{~A}$ smaller cDNA encoding this region was synthesised, cloned, and sequenced. Multiple clones had the substitution of $\mathrm{G}$ by $\mathrm{A}$ at base 2584 , converting the codon for glycine 661 to one coding for arginine (fig 2). This was confirmed by analysis of genomic DNA. Although the mutation did not destroy a restriction enzyme site, mutant DNA could be distinguished from wild type with the primer AVECO47, which contained a G instead of a C in its sequence (fig 3). After amplification this created an Eco47III restriction enzyme site when the $G$ to $A$, Gly 661 to Arg mutation was present. Analysis of the proband's DNA and that of her family confirmed that the mutation only occurred in the proband.

Testing of the type III collagen isolated from the medium showed that the secreted, overmodified form of the protein had the same thermal stability as the normal product, as both proteins were still present at $39^{\circ} \mathrm{C}$ at approximately equal intensities (fig 4A). However, the overmodified mutant intracellular protein had a reduced thermal stability, being trypsin susceptible after incubation between $37^{\circ} \mathrm{C}$ and $38^{\circ} \mathrm{C}$ (fig $4 \mathrm{~B}$ ). In both cases type I collagen acted as an internal control and melted normally.

\section{Discussion}

The proband described here has the most Nterminal glycine substitution so far described in a patient with Ehlers-Danlos syndrome type IV. Only the Gly $619^{15}$ and $136^{16}$ to Arg substitutions in patients with aortic aneurysms are more $\mathrm{N}$-terminal. The Arg 619 substitution did not affect the thermal stability of the secreted type III collagen, but a reduced thermal stability was detected when the collagenase A fragment was analysed. The secreted form of the mutant Arg 661 substitution described here also has normal thermal stability. 

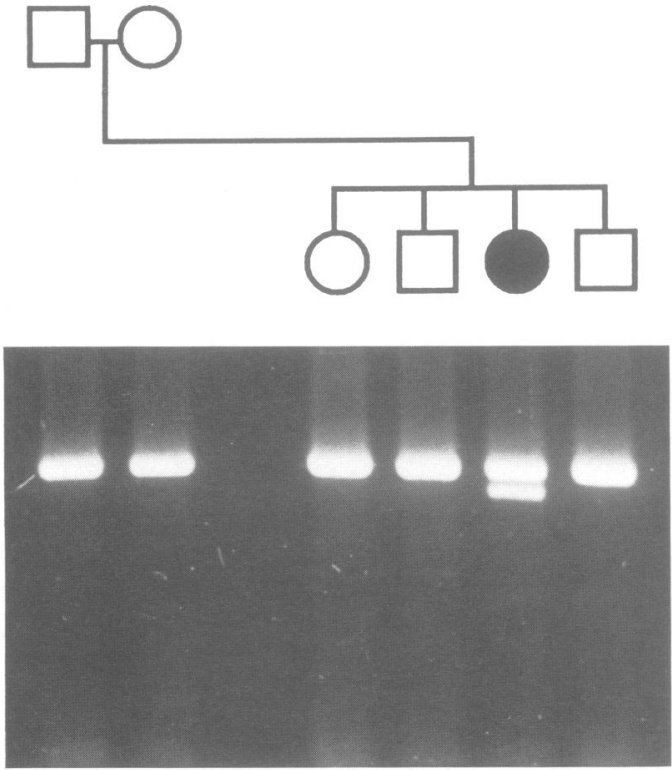

$3^{\prime}$

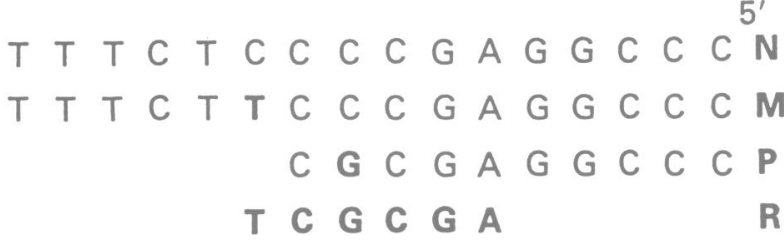

Figure 3 Family analysis. Genomic DNA was amplified then digested with Eco47III and analysed by agarose gel electrophoresis. The normal $(N)$ and mutant $(M)$ and analysed by agarose gel electrophoresis. The normal $(N)$ and mutant $(M)$ are shown, as is the $3^{\prime}$ end of the primer $A V E C O 47(P)$. The single base change in the mutant sequence is in bold type as is the mismatched base in the primer, and the recognition sequence $(R)$ of Eco47III.
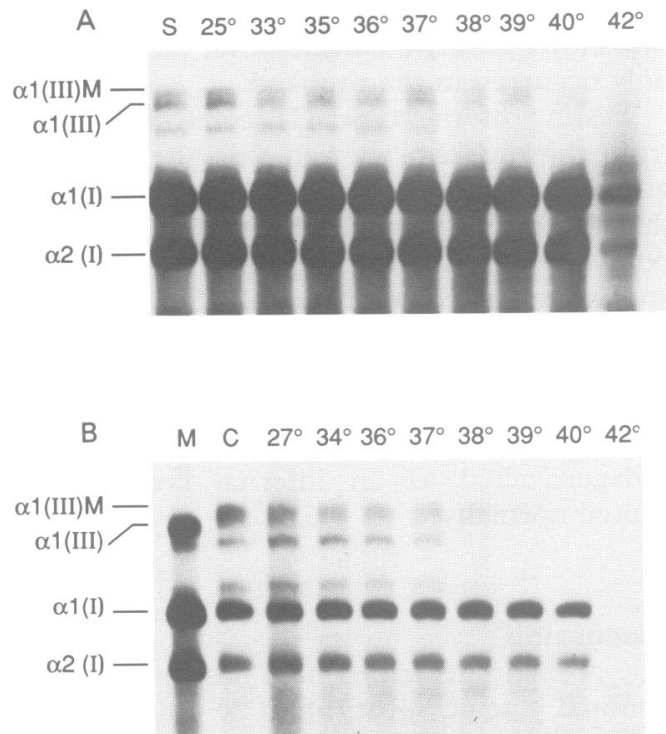

Figure 4 Thermal stability of collagens. Type III collagen secreted into the medium $(A)$ and that retained collagen secreted into the medium $(A)$ and that retained
within the cell layer $(B)$ was assayed for its sensitivity to trypsin after incubation at various temperatures, as indicated. The two forms of type III collagen present, $\alpha 1(I I I)$ and $\alpha 1(I I I) M$, are indicated. Whereas both were present in the starting material from the medium (S) only the mutant form was present in the cell layer (C) in this case. Type III collagen secreted into the medium from a normal cell line is shown for comparison (M). The two $\alpha$ chains comprising type I collagen, $\alpha 1(I)$ and $\alpha 2(I)$, are also indicated.
Interestingly, whereas the Arg 619 mutation did not affect secretion of the mutant molecule, the Arg 661 substitution caused significant intracellular retention. However, we have shown that the protein retained within the cell melted some $2^{\circ} \mathrm{C}$ lower than normal and therefore the retained molecules were those which had an altered thermal stability or were pepsin sensitive. Thus for both the Arg 619 and 661 substitutions molecules with normal thermal stability were normally secreted. This contrasts with other COL $3 \mathrm{Al}$ mutations. The Gly 910 to Val mutation is partially secreted but has a reduced thermal stability, ${ }^{17}$ while a Gly 1018 to Asp mutation has normal stability but is nearly completely retained within the cell. ${ }^{8}$ Thus, a correlation between thermal stability of these pepsin resistant triple helixes and secretion does not appear to exist.

The difference, seen here, between the secreted and retained form of the mutant protein indicates that molecules resulting from the same mutation can differ in their thermal stabilities. This is perhaps not surprising since type III collagen is a trimeric molecule and mutant forms consisting of either one, two, or three abnormal chains will exist, as will the normal product. Presumably this variable composition leads to the differences seen in thermal stability. An alternative explanation for our observations is that some normal homotrimers of type III collagen from these cells are overmodified and secreted into the medium. However, we think this is unlikely because of the asymmetrical distribution of overmodification, which is seen only in and $\mathrm{N}$ terminal of CB5 and does not affect CB9. This is consistent with the arginine substitution being present in these molecules. As yet we have no data to suggest which particular trimer composition is the more stable.

In summary the proband described here has a new dominant mutation which converts glycine 661 in type III collagen to arginine. This results in two forms of mutant protein. One is secreted and has normal thermal stability, whereas the other is retained intracellularly and melts abnormally between $37^{\circ} \mathrm{C}$ and $38^{\circ} \mathrm{C}$.

We would like to thank Dr A C Nicholls for advice on peptide mapping experiments, and Olive Cutting for technical assistance in tissue culture. We also thank the medical illustration department at the CRC for production of the figures.

1 McKusick VA. Heritable disorders of connective tissue. 4th ed. St Louis: Mosby, 1972:292-371.

2 Pope FM, Martin GR, Lichtenstein JR, et al. Patients with Ehlers-Danlos syndrome type IV lack type III collagen. Proc Natl Acad Sci USA 1975;72:1314-16.

3 Stolle CA, Pyeritz RE, Myers JC, Prockop DJ. Synthesis of an altered type III procollagen in a patient with type IV Ehlers-Danlos syndrome. F Biol Chem 1985;260:1937-44.

4 Steinmann B, Royce PM, Superti-Furga A. In: Royce PM, Steinmann B, eds. Connective tissue and its heritable disorders, molecular genetic and medical aspects. New York: Wiley Liss, 1993:351-407.

5 Bonadio J, Byers PM. Subtle structural alterations in the chains of type I procollagen produce osteogenesis imperfecta type II. Nature 1985;316:363-6.

6 Richards AJ, Ward PN, Narcisi P, Nicholls AC, Lloyd JC, Pope FM. A single base mutation in the gene for type III collagen (COL $3 A 1$ ) converts glycine 847 to glutamic acid in a family with Ehlers-Danlos syndrome type IV. An unaffected family member is mosaic for the mutation. Hum Genet 1992;89:414-18.

7 Tromp G, Kuivaniemi H, Stolle C, Pope FM, Prockop DJ. Single base mutation in the type III procollagen gene that 
converts the codon for glycine 883 to aspartate in a mild variant of Ehlers-Danlos syndrome type IV. $\mathcal{F}$ Biol Chem 1989;264:19313-17.

8 Kontusaari S, Tromp G, Kuivaniemi H, Stolle C, Pope FM, Prockop DJ. Substitution of aspartate for glycine 1018 in the type III procollagen (COL3A1) gene causes 1018 in the type III procollagen (COL $3 \mathrm{Al}$ ) gene causes type IV Ehlers-Danlos syndrome. The mutated allele is present in most blood leukocytes of the asymptomatic

9 Pope FM, Kendall BE, Slapak GI, et al. Type III collagen mutations cause fragile cerebral arteries. $\mathrm{Br} \mathcal{J}$ Neurosurg 1991;5:535-57.

10 Nicholls AC, De Paepe A, Narcisi P, et al. Linkage of a polymorphic marker for the type III collagen gene (COL3A1) to atypical autosomal dominant Ehlers-Danlos syndrome type IV in a large Belgian pedigree. Hum Genet 1988;78:276-81.

11 Richards AJ, Lloyd JC, Ward PN, De Paepe A, Narcisi P, Pope FM. Characterisation of a glycine to valine substituPope FM. Characterisation of a glycine to valine substituof type III collagen in a patient with Ehlers-Danlos of type III collagen in a patient with Ehlers-

12 Richards AJ, Lloyd JC, Narcisi P, Ward PN, Nicholls AC De Paepe A, Pope FM. A 27 bp deletion from one allele of the type III collagen gene (COL3A1) in a large family with Ehlers-Danlos syndrome type IV. Hum Genet 1992;88:325-30.

13 Narcisi $P, W u$ Y, Tromp G, et al. Single base mutation that substitutes glutamic acid for glycine 1021 in the COL $3 A 1$
gene and causes Ehlers-Danlos syndrome type IV. $\mathrm{Am} \mathcal{F}$ Med Genet 1993;46:278-83.

14 Ala-Kokko L, Kontusaari S, Baldwin CT, Kuivaniemi H, Prockop DJ. Structure of cDNA clones coding for the entire pre-proal(III) chain of human type III procollagen: differences in protein structure from type I procollagen and conservation of codon preferences. Biochem $\mathcal{F}$ 1989;260:509-16.

15 Kontusaari S, Tromp G, Kuivaniemi H, Romanic AM, Prockop DJ. A mutation in the gene for type III procollagen (COL $3 A 1)$ in a family with aortic aneurysms. $f$ Clin Invest 1990;86:1465-73.

16 Earley J, Tromp G, Kuivaniemi H, Gatalica Z, Prockop DJ. A mutation that substitutes Arg for Gly 136 in collagen III identified in a patient with fibromuscular dysplasia and an aortic aneurysm. Matrix 1993;13:34A.

17 Nuytinck L, Narcisi P, Nicholls A, Renard JP, Pope FM, De Paepe A. Detection and characterisation of an overmodified type III collagen by analysis of non-cutaneous connective tissues in a patient with Ehlers-Danlos synconnective tissues in a patient with Ehlers
drome IV. $₹$ Med Genet 1992;29:375-80. 